\title{
Efectos de la atención plena en el ámbito educativo: una revisión sistemática Gemma González-Mesa e Isaac Amigo-Vázquez*
}

Universidad de Oviedo

\begin{abstract}
Resumen: Las intervenciones basadas en atención plena en el ámbito educativo, surgen para dotar a los más jóvenes de habilidades para gestionar el estrés y mejorar en general sus habilidades socioemocionales, cognitivas y comportamentales. El objetivo del presente trabajo ha sido examinar los efectos producidos por la aplicación de estas intervenciones en estudiantes de preescolar, primaria y secundaria. Las fuentes de información fueron las bases de datos: PsycINFO, SCOPUS y WOS. Se seleccionaron doce artículos que cumpliesen los criterios de: aplicación de mindfulness en las escuelas a estudiantes y evaluación de sus efectos; diseños experimentales o cuasi-experimentales con grupo control; muestra superior a 30 participantes y programa aplicado en al menos ocho sesiones. Se producen efectos en la mayoría de las variables emocionales, psicosociales y cognitivas evaluadas a la finalización de la intervención y en el seguimiento. La atención plena se relaciona con aumento en la calidad de vida, competencia académica y social, así como reducción de problemas de internalización y externalización. Estos datos apoyan el interés de trabajar estos contenidos en los centros educativos.
\end{abstract}

Palabras clave: Atención plena, Escuela, Estudiantes, Programa.

\begin{abstract}
Effects of mindfulness in the field of education: a systematic review
Abstract: Interventions based on mindfulness in the field of education stem from the need to provide the youngest with skills to manage stress and overall, to improve their socio-emotional, cognitive and behavioral skills. The aim of the present study has been to examine the effects produced by the application of these interventions in preschool, primary and secondary students. The sources of information were the databases PsycINFO, SCOPUS and WOS. Twelve articles were selected that met the criteria of applying mindfulness in schools to students and evaluating their effects; experimental or quasi-experimental designs with control group; sample of more than 30 participants and program applied in at least eight sessions. Effects on most of the emotional, psychosocial and cognitive variables evaluated at the end of the intervention and at follow-up. Mindfulness is related to an increase in the quality of life, academic and social competence, as well as the reduction of internalization and outsourcing problems. These data support the interest of working with these contents in educational centers.
\end{abstract}

Keywords: Mindfulness, School, Students, Program.

Mindfulness se suele traducir al español como atención plena, y es la traducción al inglés de la palabra sati en lengua pali que significa conciencia, atención y recuerdo. El aspecto de "recordar" se refiere a recordar ser consciente y prestar atención, destacando

Recibido: 04/10/2017 - Aceptado: 18/12/2017 - Avance online: 22/12/2017 *Correspondencia: Isaac Amigo Vázquez.

Departamento de Psicología, Universidad de Oviedo.

C. P: 33003, Oviedo, España.

E-mail: amigo@uniovi.es la importancia de la intención en la práctica del mindfulness (Didonna, 2011).

Jon Kabat-Zinn, el pionero más importante en la aplicación terapéutica del mindfulness, lo define como "la conciencia que surge de prestar atención intencionalmente en el momento presente y sin juzgar, a las experiencias que se despliegan en cada momento"(Kabat-Zinn, 2003). Asimismo, destaca siete elementos primordiales relacionados con la actitud hacia la práctica de la atención plena: no juzgar 
(observación imparcial), paciencia (permitir que las cosas sucedan a su debido tiempo), mente de principiante (ver las cosas como si fueran la primera vez), confianza (confiar en la propia intuición), no esforzarse (meditar es no-hacer), aceptación (receptivo y abierto a sentimientos o pensamientos) y ceder o dejar ir (no apegarse a los pensamientos).

Algunos autores reconocieron dos componentes de la atención plena: un componente de autorregulación de la atención, que implica el mantenimiento de la experiencia inmediata en el momento presente, y el mantenimiento de una orientación $u$ actitud de curiosidad, apertura y aceptación (Bishop et al., 2004).

En 1979 Jon Kabat-Zinn creó el Centro Mindfulness en la Facultad de Medicina de la Universidad de Massachussets, para tratar aquellos casos y problemas clínicos que no respondían adecuadamente al tratamiento médico convencional como casos de dolor crónico y otros síntomas asociados al estrés (Vallejo, 2006). A partir de entonces, las prácticas de meditación mindfulness han demostrado ser eficaces en el tratamiento de diferentes trastornos clínicos como trastornos de alimentación (Kristeller y Hallet, 1999), ansiedad y depresión (Hofmann, Sawyer, Witt, Oh, 2010; Miller, Fletcher, Kabat-Zinn, 1995). Didonna (2011) destaca tres cualidades para aliviar estos trastornos clínicos: no juzgar, aceptación y compasión.

En el área de la Psicología se ha desarrollado y aplicado cada vez en más procesos terapéuticos. Las corrientes más actuales que utilizan intervenciones con base empírica e incorporan la práctica de mindfulness son: la Terapia de Aceptación y Compromiso (ACT; Hayes, Strosahl y Wilson, 1999), la Terapia Dialéctico-Conductual (DBT; Linehan, 2003), la Terapia Cognitiva Basada en Mindfulness (MBCT; Segal, Williams y Teasdale, 2006) y la Reducción del Estrés Basada en Mindfulness (MBSR; Kabat-Zinn, 1982).

En el ámbito educativo, en los últimos años, ha habido una creciente aceptación de que las escuelas no sólo deben proporcionar a los niños una educación formal, sino también deben considerar el bienestar de los niños (Huppert y Johnson, 2010). Las Intervenciones Basadas en la Educación Socioemocional han mostrado ser eficaces para facilitar el desarrollo de las competencias socio-emocionales y mejorar el rendimiento académico (Durlak, Weissberg, Dymnicki, Taylor y Schellinger, 2011). Recientemente, bajo el paraguas de este área, se está extendiendo la implementación de las Intervenciones Basadas en Mindfulness, Mindfulness-Based Interventions (MBI), adaptadas al contexto escolar (Felver, Celis de Hoyos, Tezanos y Singh, 2016).

Las MBI aplicadas en el contexto escolar se desarrollan con el objetivo de dotar a los más jóvenes de habilidades para gestionar el estrés y mejorar sus habilidades socio-emocionales, cognitivas y comportamentales (Felver et al., 2016). En un meta-análisis reciente se muestra que son eficaces para el incremento en las habilidades de atención, la promoción de la autorregulación ante situaciones estresantes, el incremento de la resiliencia y la disminución de los comportamientos agresivos (Zenner, Herrnleben-Kurz y Walach, 2014).

Todo ello supone la principal causa de la gran importancia que están adquiriendo a nivel educativo las habilidades mindfulness durante los últimos años en distintos países, desarrollándose diferentes proyectos. En EEUU son numerosos los estados que han incorporado la práctica del mindfulness antes y después de las clases; y en España son más de 200 los colegios públicos que ya la han incorporado, con especial incidencia en la Comunidad Autónoma de Canarias (Sanmartín, 2015).

Existe una gran diversidad de programas y medidas de resultado, y el número de estudios que evalúan las $\mathrm{MBI}$ en el entorno escolar está creciendo. Algunos de los programas de mindfulness que se aplican a los alumnos de primaria o secundaria son: el Mindfulness in Schools Project (MiSP), en Inglaterra (Modrego et al., 2016); el Inner Kids Program, MindUP, Learning Mindful Shools, Wellness Works in Schools, to Breathe y Stressed Teens, en EEUU (Mañas, Franco, Gil y Gil, 2014); Sente, iAtención Funciona!, en Brasil (Terzi et al., 2016); Compas, en Suecia; Call to CareIsrael, en Israel. Además, existen programas $\mathrm{MBI}$ exclusivos para profesores como: 
Minfulness Based Well-Education (MBWE) de la Universidad de Toronto, Cultivating Awareness and Resilience in Education (CARE) y el Stress Management and Relaxation Techniques (SMART), en EEUU. A su vez, se promueven programas de mindfulness de aplicación para alumnos y profesores de forma conjunta: el Inner Resilience Program (IRP), en EEUU; y el Programa Respira, en Colombia (Mañas et al., 2014). Así como también programas que involucran a padres y a profesores: Still Quiet Place, en EEUU; y Staf Hakeshev, en Israel (The Mindfulness Language). Encontramos incluso programas para docentes, familia y alumnos de cualquier etapa como: Treva (LópezGonzález, 2016), Aulas Felices (Arguís, Bolsas, Hernández, y Salvador, 2010) y Escuelas Conscientes, en España (Tébar, y Parra, 2015); en Brasil, MindEduca (Terzi et al., 2016).

En la presente revisión se explora el origen de los programas basados en mindfulness en el ámbito educativo y cómo se han incorporado de forma progresiva a las aulas. Se muestran varios de estos programas aplicados a estudiantes en el ámbito educativo, a nivel nacional e internacional. Se analizan los efectos de la intervención de los estudios seleccionados en tres etapas educativas; y se agrupan en base a diferentes áreas: emocional, psicosocial y cognitiva. Asimismo, se comparan sus efectos en las variables medidas según: el tipo de diseño e intervención, el grupo de control, la duración de las sesiones o los seguimientos realizados a posteriori.

A partir de lo expuesto, el objetivo del presente trabajo es examinar los efectos producidos por la aplicación de programas basados en atención plena en el ámbito educativo a estudiantes de preescolar, primaria y secundaria. Para ello, se revisarán publicaciones recientes de calidad metodológica, los programas implementados y las medidas utilizadas para evaluar su efectividad.

\section{MÉTODO}

\section{PROCEDIMIENTO}

Las fuentes de información para esta revisión fueron las bases de datos PsycINFO, Web of Science y SCOPUS. Para ello, se utilizaron las palabras clave: "mindfulness" AND "school*" AND "students" AND "program*". La búsqueda se realizó el 23 de Abril de 2017.

Los criterios para la selección de artículos fueron: que aplicasen programas de mindfulness en el ámbito educativo a estudiantes y evaluasen sus efectos; que utilizasen diseños experimentales o cuasiexperimentales con grupo control, debido a la escasa existencia de experimentales; que presentasen una muestra superior a 30 participantes, y que el programa se aplicase en al menos ocho sesiones.

La búsqueda se limitó a artículos científicos publicados en revistas con factor de impacto, así como revisiones. A su vez, se acotó el grupo de edad a: Adolescence, Childhood, Preschool Age, School Age. No hubo ninguna limitación en la fecha, ya que las investigaciones en este tema son bastante recientes, en general, son a partir del año 2010, y la mayoría, más concretamente, en los últimos 5 años. Se seleccionaron solamente artículos en inglés y español, ya que la mayoría de las publicaciones sobre esta materia se encuentran en inglés, y el español era el segundo idioma con más publicaciones.

\section{MUESTRA}

\section{PROCESO DE SELECCIÓN}

Se identificaron un total de 295 citas en las búsquedas, de estas se eliminaron 86 duplicadas. Asimismo, se excluyeron aquellos estudios que incluían a padres o profesores en la muestra; intervenciones con otro tipo de actividades físicas como el yoga, voleibol, artes marciales; u otro tipo de terapias como la Terapia Cognitivo-Conductual, la Terapia en Línea o la Terapia de Arte. Por otro lado, se incluyeron los estudios cuyos programas eran $\mathrm{MBI}$ como por ejemplo MBSR o MBCT.

En la figura 1 se muestra el diagrama de flujo del proceso de selección de la muestra de artículos revisados, según la declaración PRISMA (Urrútia y Bonfill, 2010). 


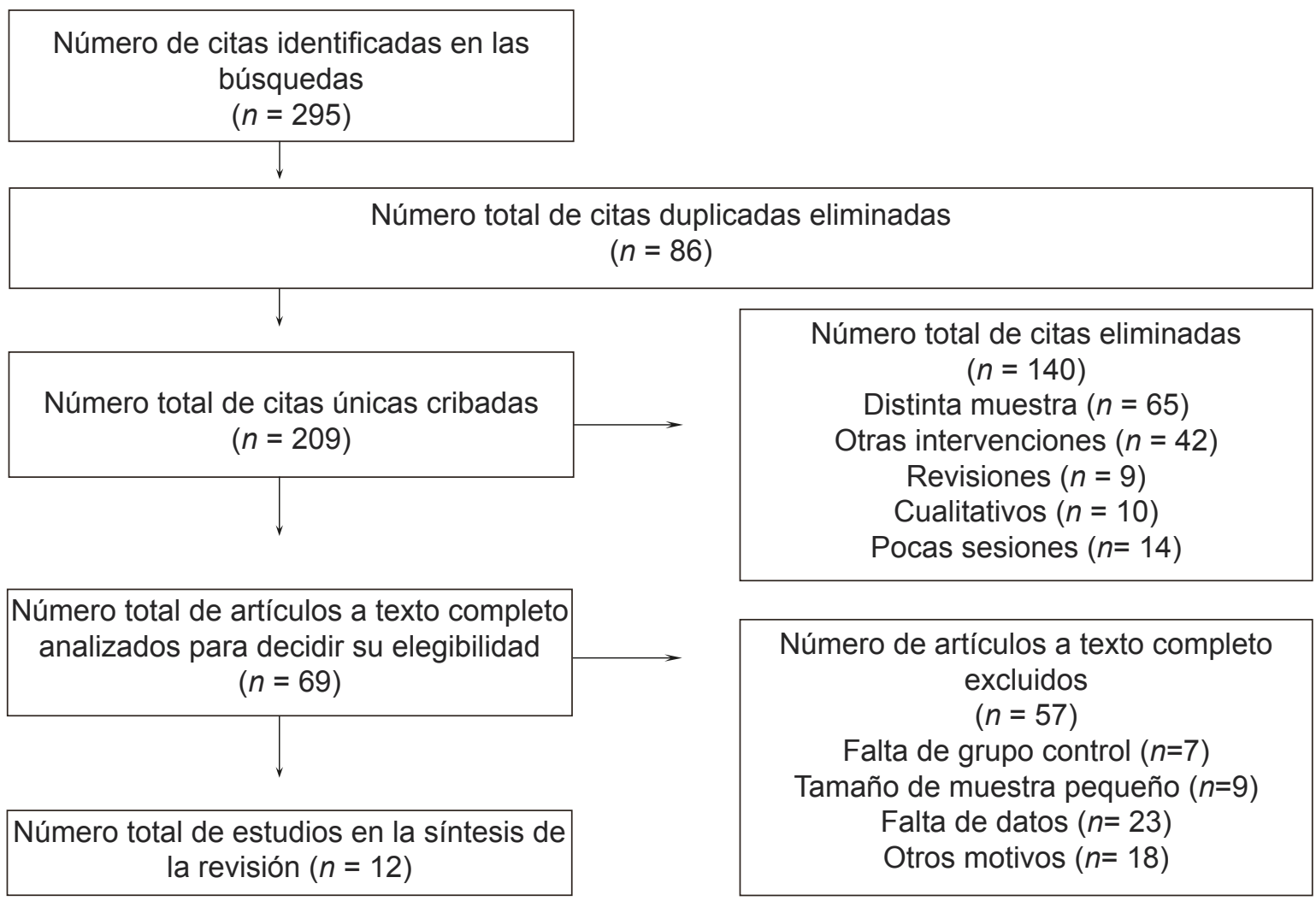

Figura 1: Proceso de selección de la muestra de artículos revisados.

\section{CARACTERÍSTICAS DE LA MUESTRA}

El rango de los años de publicación de los estudios analizados comprende desde 2013 a 2017 . Todos los artículos se publicaron en diversas revistas de habla inglesa, cada una de ellas cuenta con un artículo, a excepción de las revistas Frontiers in Psychology, Developmental Psychology y Mindfulness, que presentan dos publicaciones cada una (ver Tabla 1). A su vez, cada autor muestra una publicación.

Once de los 12 estudios informaron de alguna descripción de la edad del estudiante, el rango de edades comprende desde los 4 hasta los 17 años. De aquellos estudios que informaron de edades exactas, el promedio de la edad del estudiante fue de 10,30 años $(D T=3,80)$. Asimismo, once de los 12 estudios informaron de género. De estos aproximadamente la mitad del total de estudiantes $(49,80 \%)$ eran mujeres, lo que refleja una distribución de género bastante equilibrada. Siete de los 12 estudios informaron del nivel de grado del estudiante.
Dicho nivel fue categorizado según la clasificación de EEUU, que era el país con el mayor porcentaje de estudios (33\%). En este sistema, primaria engloba desde $1^{\circ}$ hasta $5^{\circ}$ curso y Jardín de Infancia; y secundaria desde $6^{\circ}$ a $12^{\circ}$ curso. En España primaria abarcaría hasta $6^{\circ}$ curso. Los restantes cursos de secundaria equivaldrían a la ESO a excepción de los dos últimos que sería bachillerato.

Respecto al tamaño de muestra, en los estudios experimentales varió desde 68 a 393 estudiantes $(M=187,50 ; D T=128,60)$ y en cuasi-experimentales desde 31 a 522 estudiantes $(M=194,20 ; D T=162,00)$. Un total de 2.304 estudiantes participaron en estos 12 estudios. Del total de participantes, el $67 \%$ eran estudiantes de secundaria, el $28 \%$ de primaria y un $5 \%$ de preescolar. Los programas basados en mindfulness se aplicaron a 1.251 participantes (54\%) del total, en distintos centros educativos. Las características de la muestra de los estudios revisados se exponen en la tabla 1 . 


\begin{tabular}{|c|c|c|c|c|c|}
\hline \multicolumn{6}{|c|}{$\begin{array}{c}\text { Tabla } 1 \\
\text { Características de la muestra }\end{array}$} \\
\hline Autor y año & Revista & País & Tamaño (grupos) & $\begin{array}{l}\text { Rango Edad } \\
\text { (Media) }\end{array}$ & Etapa (Curso) \\
\hline Raes et al. (2014) & Mindfulness & Bélgica & $\begin{array}{c}N=393 \\
G 1 n=194 \\
G 2 n=199\end{array}$ & $\begin{array}{c}14-17 \\
M=15.00 \\
63 \% \text { mujeres }\end{array}$ & $\begin{array}{l}\text { Secundaria } \\
\left(9^{\circ} \text { a } 12^{\circ}\right)\end{array}$ \\
\hline $\begin{array}{l}\text { Van de Weijer- } \\
\text { Bergsma et al. } \\
\text { (2014) }\end{array}$ & Mindfulness & Holanda & $\begin{array}{c}N=199 \\
G 1 n=95 \\
G 2 n=104\end{array}$ & $\begin{array}{c}8-12 \\
M=9.90 \\
55 \% \text { mujeres }\end{array}$ & $\begin{array}{l}\text { Primaria } \\
\left(3 \text { a } 5^{\circ}\right)\end{array}$ \\
\hline Flook et al. (2015) & $\begin{array}{l}\text { Developmental } \\
\text { Psychology }\end{array}$ & EEUU & $\begin{array}{c}N=68 \\
\text { G1n }=30 \\
G 2 n=38\end{array}$ & $\begin{array}{c}4-5 \\
M=4.70 \\
50 \% \text { mujeres }\end{array}$ & Preescolar \\
\hline Ricarte et al. (2015) & $\begin{array}{l}\text { International } \\
\text { Journal of } \\
\text { Cognitive Therapy }\end{array}$ & España & $\begin{array}{c}N=90 \\
\text { G1n }=45 \\
G 2 n=45\end{array}$ & $\begin{array}{c}6-13 \\
M=8.90 \\
45 \% \text { mujeres }\end{array}$ & $\begin{array}{l}\text { Primaria } \\
\left(1^{\circ} a 6^{\circ}\right)\end{array}$ \\
\hline Kuyken et al. (2013) & $\begin{array}{l}\text { The British Journal } \\
\text { of Psychiatry }\end{array}$ & Reino Unido & $\begin{array}{c}N=522 \\
G 1 n=256 \\
G 2 n=266\end{array}$ & $\begin{array}{c}12-16 \\
M=14.80 \\
29 \% \text { mujeres }\end{array}$ & Secundaria \\
\hline Metz et al. (2013) & $\begin{array}{l}\text { Research in Human } \\
\text { Development }\end{array}$ & EEUU & $\begin{array}{c}N=216 \\
G 1 n=148 \\
G 2 n=95\end{array}$ & $\begin{array}{c}M=16.40 \\
61 \% \text { mujeres }\end{array}$ & $\begin{array}{l}\text { Secundaria } \\
\left(10^{\circ} \text { a } 12^{\circ}\right)\end{array}$ \\
\hline Viafora et al. (2015) & $\begin{array}{l}\text { Journal of child } \\
\text { and family studies }\end{array}$ & EEUU & $\begin{array}{c}N=63 \\
\text { G1n }=28 \\
G 2 n=15 \\
G 3 n=20\end{array}$ & $\begin{array}{c}11-13 \\
M=11.80\end{array}$ & $\begin{array}{l}\text { Secundaria } \\
\left(6^{\circ} \text { a } 8^{\circ}\right)\end{array}$ \\
\hline $\begin{array}{l}\text { Schonert-Reichl et } \\
\text { al. (2015) }\end{array}$ & $\begin{array}{l}\text { Developmental } \\
\text { Psychology }\end{array}$ & Canadá & $\begin{array}{c}N=99 \\
\text { G1n }=48 \\
G 2 n=51\end{array}$ & $\begin{array}{c}9-11 \\
M=10.00 \\
44 \% \text { mujeres }\end{array}$ & $\begin{array}{l}\text { Primaria } \\
\left(4^{\circ}, 5^{\circ}\right)\end{array}$ \\
\hline $\begin{array}{l}\text { Crescentini et al. } \\
(2016)\end{array}$ & $\begin{array}{l}\text { Frontiers in } \\
\text { Psychology }\end{array}$ & Italia & $\begin{array}{c}N=31 \\
\text { G1n }=16 \\
\text { G2n }=15\end{array}$ & $\begin{array}{c}7-8 \\
M=7.30 \\
51 \% \text { mujeres }\end{array}$ & Primaria \\
\hline $\begin{array}{l}\text { Terjestam et al. } \\
\text { (2016) }\end{array}$ & $\begin{array}{l}\text { School Psychology } \\
\text { International }\end{array}$ & Suecia & $\begin{array}{c}N=358 \\
\text { G1n }=215 \\
G 2 n=143\end{array}$ & $48 \%$ mujeres & $\begin{array}{l}\text { Secundaria } \\
\left(5^{\circ}, 7^{\circ}, 8^{\circ}\right)\end{array}$ \\
\hline Thierry et al. (2016) & $\begin{array}{l}\text { Early Education } \\
\text { and Develpment }\end{array}$ & EEUU & $\begin{array}{c}N=47 \\
G 1 n=23 \\
G 2 n=24\end{array}$ & $\begin{array}{c}M=4.50 \\
50 \% \text { mujeres }\end{array}$ & Preescolar \\
\hline $\begin{array}{l}\text { Tarrasch et al. } \\
\text { (2017) }\end{array}$ & $\begin{array}{l}\text { Frontiers in } \\
\text { Psychology }\end{array}$ & Israel & $\begin{array}{l}N=218 \\
G 1 n=138 \\
G 2 n=78\end{array}$ & $\begin{array}{c}9-10 \\
M=10.40 \\
49 \% \text { mujeres }\end{array}$ & $\begin{array}{l}\text { Primaria } \\
\left(4^{\circ}, 5^{\circ}\right)\end{array}$ \\
\hline
\end{tabular}




\section{DISEÑO DE LA INVESTIGACIÓN}

Solo cuatro de los 12 estudios realizaron un diseño controlado aleatorizado a nivel individual, los ocho restantes utilizaron un diseño cuasi-experimental. Todos los estudios presentan grupo control y sus características han sido distintas según el estudio. El tipo de grupo control predominante en los artículos fue participantes en lista de espera, en siete de ellos. En los cinco restantes se empleó grupo de control activo; a sus participantes, según el estudio, se les proporcionó una Instrucción Regular Musical (IMR), un Programa de Responsabilidad Social (BAU), Conocimiento Emocional (CE) o Lecciones Académicas Grabadas (LAG).

Tabla 2

Características de los estudios controlados aleatorizados

\begin{tabular}{|c|c|c|c|c|}
\hline Autor & Intervención & Medidas & Resultados & Conclusión \\
\hline Raes et al. & $\begin{array}{l}\text { G1: MBI } \\
\text { G2: LE } \\
\text { MBI:MBSR } \\
+\mathrm{MBCT} \\
+15 \text { min/día práctica } \\
\text { casa } \\
8 \text { sesiones } \\
\text { 1/semana } \\
\text { (100 min.) }\end{array}$ & $\begin{array}{l}\text { Tiempos: } \\
\text { T1, T2, T3 } \\
\text { (6 meses) } \\
\text { Medidas: } \\
\text { DASS-21 }\end{array}$ & $\begin{array}{l}\text { G1 en T2 y T3 } \\
<\text { DASS-21 }\end{array}$ & $\begin{array}{l}\text { MBI reduce niveles } \\
\text { de depresión hasta } 6 \\
\text { meses después }\end{array}$ \\
\hline $\begin{array}{l}\text { Van de Weijer-Bergsma } \\
\text { et al. }\end{array}$ & $\begin{array}{l}\text { G1: MK } \\
\text { G2: LE } \\
\text { MK: MBSR } \\
+ \text { MBCT } \\
\text { + práctica opcional } \\
\text { casa } \\
12 \text { sesiones } \\
\text { 2/semana } \\
\text { (30 min.) }\end{array}$ & $\begin{array}{l}\text { Tiempos: } \\
\text { T1, T2, T3 (7semanas) } \\
\text { Medidas: } \\
\text { NPDK } \\
\text { EAQ-30 } \\
\text { SOC-K, SHS } \\
\text { SCARED-71, SCBE-30 } \\
\text { SDSC } \\
\text { SCCPII }\end{array}$ & $\begin{array}{l}\text { G1 en T2, T3: } \\
\text { < NPDK, } \\
\text { > EAQ-30, SHS } \\
\text { G1 en T2: } \\
\text { > SCBE-30, PCCS II } \\
\text { G1 enT3: } \\
\text { < SCARED71 }\end{array}$ & $\begin{array}{l}\text { MK produce efectos } \\
\text { inmediatos y a largo } \\
\text { plazo en la salud } \\
\text { mental y emocional de } \\
\text { los niños. } \\
\text { Menos rumia y } \\
\text { ansiedad, mayor } \\
\text { competencia social. }\end{array}$ \\
\hline Flook et al. & $\begin{array}{l}\text { G1: KC } \\
\text { G2: LE } \\
\text { KC: MBI } \\
\text { + lecturas } \\
\text { + música y movimiento } \\
24 \text { sesiones } \\
\text { 2/semana } \\
\text { (20/30 min) }\end{array}$ & $\begin{array}{l}\text { Tiempos } \\
\text { T1 y T2 } \\
\text { Medidas } \\
\text { TSC, TS } \\
\text { GD, TF } \\
\text { DCCS } \\
\text { GS }\end{array}$ & $\begin{array}{l}\text { G1 en T2: } \\
>\text { TSC, TS, GS, DCCS }\end{array}$ & $\begin{array}{l}\text { KC mejora el } \\
\text { comportamiento pro- } \\
\text { social, la regulación } \\
\text { emocional, el } \\
\text { aprendizaje y la } \\
\text { flexibilidad cognitiva. }\end{array}$ \\
\hline Ricarte et al. & $\begin{array}{l}\text { G1: MBI } \\
\text { G2: LE } \\
\text { MEITP } \\
\text { + atención visual y } \\
\text { auditiva } \\
30 \text { sesiones } \\
\text { 1/día } \\
\text { (15 min.) }\end{array}$ & $\begin{array}{l}\text { Tiempos } \\
\text { T1 y T2 } \\
\text { Medidas } \\
\text { STAIC } \\
\text { TMT } \\
\text { Faces-R } \\
\text { WISC-III }\end{array}$ & $\begin{array}{l}\text { G1 en T2: } \\
<\text { STAIC } \\
>\text { dígitos adelante en } \\
\quad \text { WISC-III }\end{array}$ & $\begin{array}{l}\text { MBI disminuye sig. } \\
\text { niveles AR. Mejora } \\
\text { memoria auditivo- } \\
\text { verbal inmediata }\end{array}$ \\
\hline
\end{tabular}

Nota: $\mathrm{MBI}=$ Intervención Basada en Atención Plena; $\mathrm{MBSR}=$ Reducción de Estrés Basada en la Atención Plena; $\mathrm{MBCT}=$ Terapia Cognitiva Basada en Mindfulness; $\mathrm{MK}=\mathrm{Mindfulkids;} \mathrm{KC}=$ Kindness Curriculum; MEITP= Mindfulness Emotional Intelligence Training Program; G1 = Grupo 1; G2= Grupo 2; G3= Grupo 3; $L E=$ lista de espera; $T 1=$ medida pre-test; $T 2=$ medida pos-test; T3= medida de seguimiento. (Ver escalas en Apéndice). 
Tabla 3

Características de los estudios cuasi-experimentales

\begin{tabular}{|c|c|c|c|c|}
\hline Autor y año & Intervención & Medidas & Resultados & Conclusión \\
\hline Kuyken et al. & $\begin{array}{l}\text { G1: MISP } \\
\text { G2: LE } \\
\text { MISP: MBSR } \\
\text { + MBCT } \\
9 \text { sesiones } \\
\text { 1/semana }\end{array}$ & $\begin{array}{l}\text { Tiempos: } \\
\text { T1, T2, T3 (3 meses) } \\
\text { Medidas: QD } \\
\text { WEMWBS } \\
\text { PSS, QEF } \\
\text { CES-D }\end{array}$ & $\begin{array}{l}\text { Glen T2: } \\
\text { < PSS, > QEF } \\
\text { G1:T2 y T3 } \\
\text { < CES-D, > WEMWBS }\end{array}$ & $\begin{array}{l}\text { MISP Mejora estrés, } \\
\text { síntomas depresivos y } \\
\text { bienestar hasta } 3 \text { meses } \\
\text { después. }\end{array}$ \\
\hline Metz et al. & $\begin{array}{l}\text { G1: LB } \\
\text { G2: IMR } \\
\text { LB: MBSR } \\
+ \text { CDs casa } \\
18 \text { sesiones } \\
1 / \text { semana } \\
(15 / 25 \mathrm{~min})\end{array}$ & $\begin{array}{l}\text { Tiempos: } \\
\text { T1 y T2 } \\
\text { Medidas: } \\
\text { DERS, QD } \\
\text { PCS, SS, ASRES } \\
\text { SAVPS }\end{array}$ & $\begin{array}{l}\text { G1 en T2: } \\
\text { > DERS, ASRES, } \\
\text { SAVPS } \\
\text { < PCS, SS }\end{array}$ & $\begin{array}{l}\text { LB mejora la regulación } \\
\text { emocional, el nivel } \\
\text { de estrés y síntomas } \\
\text { psicosomáticos. }\end{array}$ \\
\hline Viafora et al. & $\begin{array}{l}\text { G1: MBI } \\
\text { G2: MBI } \\
\text { G3: LE } \\
\text { MBI: MBSR } \\
8 \text { sesiones } \\
\text { 1/semana } \\
\text { (45 min.) }\end{array}$ & $\begin{array}{l}\text { Tiempos } \\
\text { T1 y T2 } \\
\text { Medidas } \\
\text { CAMM } \\
\text { AFQ-Y } \\
\text { SCS-C } \\
\text { QD, PEQ }\end{array}$ & $\begin{array}{l}\text { En T2: } \\
\text { G1cambio sig. CAMM } \\
\text { G2 > PEQ }\end{array}$ & $\begin{array}{l}\text { MBI aumenta la } \\
\text { atención plena en } \\
\text { estudiantes con hogar. }\end{array}$ \\
\hline Schonert-Reichl et al. & $\begin{array}{l}\text { G1: MUP } \\
\text { G2: BAU } \\
\text { MUP: MBI + SEL } \\
\text { + literatura } \\
\text { + autoreflexión } \\
12 \text { sesiones } \\
\text { 1/semana } \\
\text { (40/45 min) }\end{array}$ & $\begin{array}{l}\text { Tiempos } \\
\text { T1 y T2 } \\
\text { Medidas } \\
\text { TF, TFH } \\
\text { CS, QD, IRI, RI, SDQ } \\
\text { SPQC, MG, MAAS-C } \\
\text { SGQ, MP }\end{array}$ & $\begin{array}{l}\text { G1 en T2: } \\
\text { < TR y más precisión en } \\
\text { TF y TFH. } \\
\text { Mejoras sig. en IRI, RI, } \\
\text { SDQ, SPQC, MAASC } \\
\text { > rendimiento } \\
\text { matemático. }\end{array}$ & $\begin{array}{l}\text { MUP mejora EFs, } \\
\text { empatía, control } \\
\text { emocional, } \\
\text { auto-concepto, } \\
\text { atención plena y } \\
\text { comportamiento } \\
\text { prosocial. } \\
\text { Reduce síntomas } \\
\text { depresivos y estrés. }\end{array}$ \\
\hline Crescentini et al. & $\begin{array}{l}\text { G1: MOM } \\
\text { G2: CE } \\
\text { MOM: MBSR } \\
\text { 24 sesiones } \\
\text { 3/semana } \\
\text { (30-90 min) }\end{array}$ & $\begin{array}{l}\text { Tiempos } \\
\text { T1 y T2 } \\
\text { Medidas } \\
\text { CBCL-TRF } \\
\text { CTRS-R } \\
\text { SMFQ }\end{array}$ & $\begin{array}{l}\text { G1 en T2: mejoras } \\
\text { índice TDAH e } \\
\text { impulsividad. } \\
\text { G1 y G2 en T2: } \\
\text { < problemas } \\
\text { internalización, } \\
\text { externalización. }\end{array}$ & $\begin{array}{l}\text { MOM aumenta } \\
\text { atención. } \\
\text { Ambos grupos reducen } \\
\text { ansiedad, depresión y } \\
\text { quejas psicosomáticas. }\end{array}$ \\
\hline Terjestam et al. & $\begin{array}{l}\text { G1: Compas } \\
\text { G2: LAG } \\
\text { Compas: } \\
\text { Grabaciones MBI } \\
\text { + RM } \\
24 \text { sesiones } \\
3 / \text { semana } \\
\text { (22 min.) }\end{array}$ & $\begin{array}{l}\text { Tiempos } \\
\text { Tl y T2 } \\
\text { Medidas } \\
\text { EATO-R: } \\
\text { EC } \\
\text { WBS, PD } \\
\text { GSS } \\
\text { SDQPP }\end{array}$ & $\begin{array}{l}\text { G1 en T2: } \\
\text { >EC, WBS, SDQPP }\end{array}$ & $\begin{array}{l}\text { Compas mejora la } \\
\text { atención, inhibición, } \\
\text { bienestar y relaciones } \\
\text { sociales. }\end{array}$ \\
\hline Thierry et al. & $\begin{array}{l}\text { G1: MUP } \\
\text { G2: BAU } \\
\text { MUP: MBI } \\
15 \text { sesiones } \\
\text { 3/día } \\
\text { (20/30 min) } \\
7 \text { meses }\end{array}$ & $\begin{array}{l}\text { Tiempos } \\
\text { T1 y T2 } \\
\text { Medidas } \\
\text { BRIEF-P } \\
\text { PPVT-4 } \\
\text { ISIP }\end{array}$ & $\begin{array}{l}\text { G1 en T2: } \\
\text { > ISIP, mejoras sig. en } \\
\text { memoria de trabajo y } \\
\text { organización. }\end{array}$ & $\begin{array}{l}\text { MUP regula los niveles } \\
\text { en funciones ejecutivas. } \\
\text { Mejora el aprendizaje } \\
\text { de vocabulario. }\end{array}$ \\
\hline Tarrasch et al. & $\begin{array}{l}\text { G1: C2C-I } \\
\text { G2: LE } \\
\text { C2C-I: MBI } \\
24 \text { sesiones } \\
\text { 1/semana } \\
\text { (45 min.) }\end{array}$ & $\begin{array}{l}\text { Tiempos } \\
\text { T1 y T2 } \\
\text { Medidas } \\
\text { VMI: VP, MC } \\
\text { SCAS, FFMQ }\end{array}$ & $\begin{array}{l}\text { G1 en } T 1 \text { > VMI: niñas, } \\
\text { SCAS, FFMQ }\end{array}$ & $\begin{array}{l}\text { C2C-I aumenta } \\
\text { precisión visual y } \\
\text { motora, la atención } \\
\text { plena y reduce niveles } \\
\text { ansiedad. }\end{array}$ \\
\hline
\end{tabular}




\section{RESULTADOS}

Se analizaron 12 artículos que cumplían los criterios anteriormente especificados, que abordan la eficacia de los programas de mindfulness en estudiantes de preescolar, primaria y secundaria.

Las características principales de los estudios se exponen en las tablas 2 y 3.

\section{CARACTERÍSTICAS DE LA INTERVENCIÓN}

\section{TIPOS DE PROGRAMAS}

El programa básico de Reducción de Estrés Basado en Mindfulness (MBSR) consiste en ocho semanas con prácticas de dos a tres horas diarias, y finaliza con un encuentro que dura todo un día (Hick y Bien, 2008). El programa incluye prácticas formales e informales. Las prácticas informales enfatizan el mindfulness en la vida cotidiana (Baer, 2003) con la toma de conciencia a las actividades diarias como comer. Las prácticas formales implican dedicar un tiempo para permanecer sentado, tumbado o de pie, y centrar la atención en la respiración (Vallejo, 2006); junto con determinados ejercicios de relajación como la exploración del cuerpo o body scan (Kabat-Zinn, 2003), que consiste en una mera experimentación de las sensaciones corporales asociadas al repaso activo del cuerpo y movimientos conscientes.

El tipo de intervención implementada en seis de los 12 estudios se fundamentó en el Programa MBSR, y en tres de ellos se utilizó además la MBCT. Estos programas se han adaptado, en general, al grupo de edad. En los restantes estudios se han realizado diferentes adaptaciones de varios tipos de programas. El Programa Kindness Curriculum $(K C)$, que es un entrenamiento de habilidades prosociales basado en mindfulness, se aplicó en el estudio de Flook, Goldberg, Pinger, y Davidson (2015). Una adaptación del Programa de Mindfulness de entrenamiento en Inteligencia Emocional (Ramos, Recondo y Enríquez, 2012) se empleó en el estudio de Ricarte, Ros, Latorre y Beltrán (2015). El método MindUP, basado en el Programa SEL
(Social and Emotional Learning) de educación basada en mindfulness, se implementó en los estudios de Schonert-Reichl et al. (2015); y Thierry, Bryant, Nobles y Norris, (2016). El Programa Compas (Compassion and Attencion in the Schools) que combina mindfulness $y$ Aprendizaje Socio-Emocional, se aplicó en el estudio de Terjestam, Bengtsson y Jansson (2016). Call to Care-Israel se basa en dos programas, uno que cultiva la compasión, del Instituto de Mente y Vida (Charlottesville, VA, USA); y otro Programa Socio-Emocional de mejora de la resiliencia para estudiantes que viven con estrés (ERASE-S), se ha aplicado en el estudio de Tarrasch, Margalit-Shalom y Berger (2017).

\section{FORMATO Y DURACIÓN DE LA INTERVENCIÓN}

La mitad de las $\mathrm{MBI}$ en los estudios fueron impartidas por un facilitador externo experimentado, y la otra mitad por profesores capacitados. Las MBI se aplicaron, según el estudio, entre las 8 y las 30 sesiones ( $M$ $=19,33 ; D T=7,34)$ con una duración comprendida entre 15 y 100 minutos por sesión ( $M=44,00 ; D T=22,70)$. Un solo estudio no recoge la duración de las sesiones (Kuyken et al., 2013). La frecuencia de las sesiones mayoritaria era de una sesión por semana, en siete de los estudios.

\section{CARACTERÍSTICAS DE LOS RESULTADOS}

\section{TIEMPOS DE EVALUACIÓN}

En los 12 estudios, las medidas de evaluación se tomaron en dos tiempos diferentes: pre-tratamiento y pos-tratamiento. $Y$ en tres estudios además, se tomaron medidas de seguimiento. Estas fueron, según el estudio: a las 7 semanas, a los 3 y 6 meses después de la finalización de la intervención.

\section{ESCALAS}

Los 12 estudios presentan una amplia variabilidad de escalas para evaluar las diferentes medidas, tan solo dos artículos utilizan la misma prueba para medir el control 
inhibitorio y la flexibilidad cognitiva, esta ha sido: TF (Flanker task; Davidson, Amso, Anderson y Diamond, 2006).

\section{VARIABLES ANALIZADAS}

Las variables más analizadas en la totalidad de los estudios han sido: la competencia social, la regulación emocional y la flexibilidad cognitiva (ver figura 2). A continuación, se detallan sus escalas.

La competencia social se ha evaluado en cinco de los 12 artículos, con ocho escalas diferentes: SCBE-30 (Social Competence and Behavior Evaluation; LaFreniere y Dumas, 1996), TSC (Teacher Social Competence Scale; Conduct Problems Prevention Research Gruop, 1995), SDQPP (Strengths and Difficulties Questionnaire; Goodman, Meltzer y Bailey, 1998), CBCL-TRF (Child Behavior Checklist-Teacher Report Form; Achenbach y Dumenci, 2001), SGQ (Social Goals Questionnaire; Wentzel, 1993), MP (Peer-Report Measures; Parkhurst y Asher, 1992), TS (Sharing Task; Flook et al., 2015), SCCP II (School as a Caring Community Profile II; Lickona y Davidson, 2003).

La regulación emocional se evaluó en cinco de los 12 artículos con seis escalas diferentes: DERS (Difficulties in Emotion Regulation Scale; Gratz y Roemer, 2004), ASRES (Affective SelfRegulatory Efficacy Scale; Bandura, 2006); EAQ-30 (Emotion Awareness Questionnaire; Rieffe, Oosterveld, Miers, Meerum y Ly, 2008), TSC (Teacher Social Competence Scale; Conduct Problems Prevention Research Group, 1995), Rl (Resiliency Inventory; Noam y Golstein, 1998) y BRIEF-P (Inventario de Evaluación Conductual de la Función Ejecutiva-Preescolar; Gioia, Espy y Isquith, 2002).

La flexibilidad cognitiva se ha evaluado en cinco de los 12 artículos con seis escalas diferentes: AFQ-Y (The Avoidance and Fusion Questionnaire for Youth; Greco, Lambert y Baer, 2008), DCCS (Dimensional Change Card Sort Task; Garon, Bryson y Smith, 2008), TMT (Trail Making Test; Reitan, 1992), TF, TFH (Hearts and Flowers Task; Diamond, Barnett, Thomas y Munro, 2007) y BRIEF-P.
Cabe decir que la atención y la depresión, aunque se evaluaron en menor número de artículos, se utilizaron un total de seis y cinco escalas diferentes, respectivamente. A su vez, las siguientes variables se han evaluado cada una, en uno de los 12 estudios: autocompasión, auto-concepto, empatía, impulsividad, integración viso-motriz y memoria auditivo-verbal inmediata.

De todas las variables analizadas, un $67 \%$ fueron auto-reportadas por los niños, un $24 \%$ por los maestros y un $8 \%$ por los padres. Los estudios que incluyeron múltiples informantes fueron el $41 \%$ del total.

\section{EFECTOS DE LA INTERVENCIÓN}

La descripción de los efectos se ha organizado en tres áreas temáticas, según el tipo de variable medida: (a) emocionales, entre ellas depresión, ansiedad, estrés y regulación de emociones; (b) psicosociales, como el bienestar psicológico, atención plena, competencia social, desempeño académico, aceptabilidad, auto-concepto y autocompasión; (c) cognitivas, entre ellas la atención, quejas psicosomáticas, rumia, impulsividad, empatía, integración visomotriz, memoria auditivo-verbal inmediata, las funciones ejecutivas como la inhibición, flexibilidad cognitiva y memoria de trabajo.

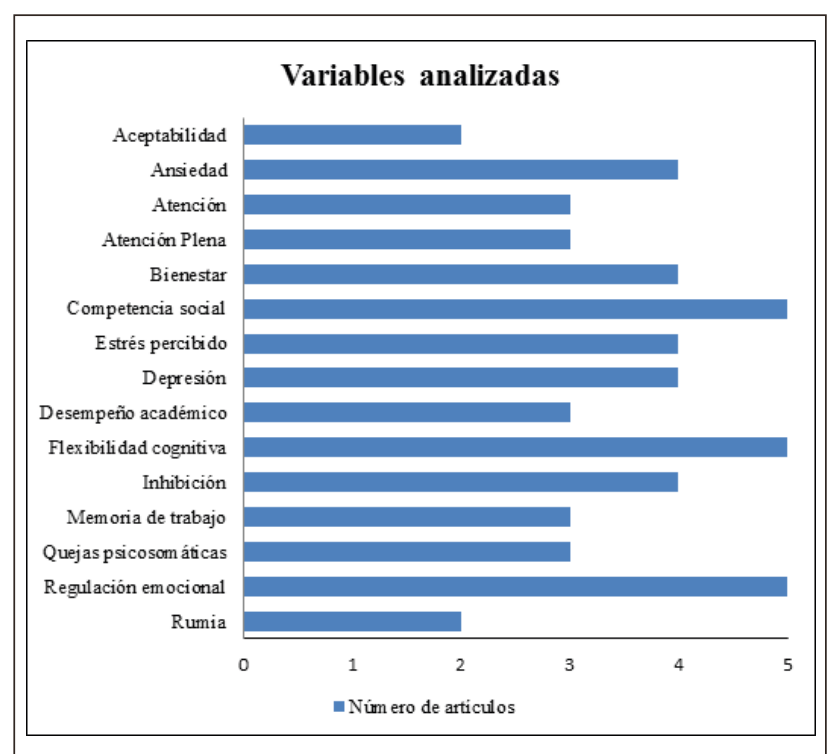

Figura 2: Variables analizadas más frecuentes en los artículos revisados. 


\section{EFECTOS EN VARIABLES EMOCIONALES}

En tres de los cuatro estudios que evaluaban la depresión, se concluyó una reducción significativa de sus síntomas en el grupo de intervención en mindfulness, con respecto al grupo control. Un estudio de primaria (Schonert-Reichl et al., 2015) confirmó dicha reducción al finalizar la intervención, y dos de secundaria que realizaron seguimiento mostraron el mantenimiento del efecto hasta 3 meses (Kuyken et al., 2013) y 6 meses después (Raes et al., 2014). Este último fue el único con diseño experimental.

En relación con el estrés, tres de los cuatro estudios cuasi-experimentales que medían esta variable, encontraron que se producía una reducción significativa en el grupo experimental respecto del grupo control. Dos en secundaria (Kuyken et al., 2013; Metz et al., 2013) y uno en primaria (Schonert-Reichl et al., 2015). Este último encontró diferencias significativas en la medición de cortisol salivar a primera hora de la mañana. Todos los estudios, excepto el de Kuyken et al. (2013), utilizaron grupo de control activo.

Tres de los cuatro estudios de primaria que midieron la ansiedad, concluyeron que las $\mathrm{MBI}$ disminuían significativamente sus niveles respecto a los grupos control. Dos de ellos (Tarrasch et al., 2017; Ricarte et al., 2015) mostraron esta disminución en el grupo experimental al finalizar la intervención, y uno de ellos (Van de Weijer-Bergsma, Langenberg, Brandsma, Oort y Bögels, 2014) constató una mayor reducción en el momento del seguimiento a las 7 semanas. Solo este último y Ricarte et al. (2015) utilizaron diseños controlados aleatorizados.

Respecto a las habilidades de regulación emocional, se observó un aumento significativo en el grupo experimental en cuatro de los cinco estudios que evaluaban esta medida respecto a los controles. Uno de ellos en secundaria (Metz et al., 2013), otro en preescolar (Flook et al., 2015) y dos en primaria (Schonert-Reichl et al., 2015; Van de Weijer-Bergsma et al., 2014). Este último fue el único que hizo seguimiento, y mostró mejoras significativas tanto al finalizar la intervención como a las 7 semanas. Dicho estudio, junto con Flook et al. (2015), utilizaron diseños experimentales.

\section{EFECTOS EN VARIABLES PSICOSOCIALES}

Los cuatro estudios que evaluaron el bienestar psicológico, mostraron un aumento significativo en el grupo experimental en relación al grupo control. Dos en secundaria (Kuyken et al., 2013; Terjestam et al., 2016) y dos en primaria (Schonert-Reichl et al., 2015; Van de Weijer-Bergsma et al., 2014). Terjestam et al. (2016) encontraron diferencias solamente en el grupo de $5^{\circ}$ grado. Y los dos que realizaron seguimiento mostraron mejoras a los 3 meses (Kuyken et al., 2013) y a las 7 semanas (Van de Weijer-Bergsma et al., 2014). Este último fue el único con diseño experimental.

En cuanto a la medida de atención plena, los tres estudios cuasi-experimentales que midieron esta variable, encontraron aumentos significativos en el grupo de intervención en mindfulness en comparación con el grupo control. Dos en primaria (Schonert-Reichl et al., 2015; Tarrasch et al., 2017) y uno en secundaria (Viafora, Mathiesen y Unsworth, 2015). Este último mostró dichos aumentos en el grupo de estudiantes que tenían hogar.

Respecto a la competencia social, en cuatro de los cinco estudios que la evaluaron se produjeron cambios significativos en el grupo experimental al finalizar la intervención en comparación al grupo control. Uno en preescolar (Flook et al., 2015), dos en primaria (Schonert-Reichl et al., 2015; Van de WeijerBergsma et al., 2014) y uno en secundaria (Terjestam et al., 2016). Van de WeijerBergsma et al. (2014) y el estudio de Flook et al. (2015) realizaron diseños experimentales.

En relación con el desempeño académico, los tres estudios que evaluaron esta medida encontraron calificaciones más altas en el grupo experimental tras la intervención en algunas materias, respecto a sus controles. Uno en primaria (Schonert-Reichl et al., 2015) en matemáticas. $Y$ otros dos en preescolar: Flook et al. (2015) en el enfoque de aprendizaje, temas de salud, desarrollo social y emocional; y Thierry et al. (2016) en lectura, escritura y vocabulario. Flook et al. (2015) fue el único con diseño experimental.

Dos estudios cuasi-experimentales en secundaria (Kuyken et al., 2013 y Metz et al., 
2013) mostraron una alta aceptabilidad de las $\mathrm{MBI}$ en los estudiantes. Asimismo, un estudio cuasi-experimental en primaria (SchonertReichl et al., 2015) evaluó la medida de autoconcepto y concluyó mejoras significativas en el grupo experimental con respecto al grupo activo.

\section{EFECTOS EN VARIABLES COGNITIVAS}

Dos de los tres estudios que evaluaron la atención, encontraron diferencias significativas en el grupo experimental en comparación con el grupo control activo tras la intervención. Uno en secundaria (Teriestam et al., 2016) y otro en primaria (Crescentini et al., 2016); ambos cuasi-experimentales. Respecto a las quejas psicosomáticas, solo uno de los tres estudios cuasi-experimentales que evaluaron esta medida, mostró diferencias significativas entre los grupos (Metz et al., 2013) en secundaria, tras la intervención, respecto a sus controles.

Un estudio experimental (Van de WeijerBergsma et al., 2014), de los dos que evaluaron la rumia en primaria, encontró una disminución significativa en el grupo de intervención en mindfulness a la finalización de la intervención y en el seguimiento a las 7 semanas, en comparación con el grupo control. Asimismo, las siguientes variables: impulsividad, empatía, integración viso-motriz y memoria auditivo-verbal inmediata; se evaluaron cada una de ellas en los estudios de primaria de Crescentini et al. (2016), SchonertReichl et al. (2015), Tarrasch et al. (2017) y Ricarte et al. (2015), respectivamente, y se encontraron diferencias significativas en todas las medidas tras la intervención, en relación con los grupos control. Dos de los estudios utilizaron grupo de control activo y los otros dos en lista de espera. El estudio de Ricarte et al., (2015) era el único experimental.

En cuanto a las funciones ejecutivas se encontró que tras la intervención: (a) en flexibilidad cognitiva, dos de los cinco artículos que la evaluaron mostraron diferencias significativas entre los grupos, uno experimental en preescolar (Flook et al., 2015) y otro cuasi-experimental en secundaria (Schonert-Reichl et al., 2015); (b) en la medida de inhibición, dos de los cuatro artículos que la evaluaron, encontraron diferencias significativas entre los grupos, uno en primaria (Schonert-Reichl et al., 2015) y otro en secundaria (Terjestam et al., 2016), ambos cuasi-experimentales y con grupo de control activo; (c) en la memoria de trabajo, dos de los tres estudios que evalúan dicha medida, mostraron un aumento significativo en el grupo experimental respecto al grupo control; uno en preescolar (Thierry et al., 2016) y otro en secundaria (Schonert-Reichl et al., 2015), ambos también con diseños cuasi-experimentales y con grupos de control activos.

\section{DISCUSIÓN}

El objetivo del presente trabajo ha sido examinar los efectos producidos por la aplicación de programas basados en atención plena en el ámbito educativo a estudiantes de preescolar, primaria y secundaria. Se observan efectos beneficiosos en la regulación emocional, la competencia social y las funciones ejecutivas en todas las etapas educativas. En la etapa de primaria destacan además la ansiedad, la depresión, la atención plena, el desempeño académico y la atención cognitiva. En secundaria a su vez, la depresión, el estrés, el bienestar psicológico, la atención plena y la atención cognitiva. Asimismo, en preescolar el desempeño académico.

Los datos indican que la reducción de la ansiedad se produce en la mayoría de los estudios que la evaluaron con independencia del tipo de diseño y programa de intervención en mindfulness, así como el tiempo y la duración de las sesiones. Dicha mejoría se produce como consecuencia de prestar atención a la respiración; observar con interés y curiosidad pensamientos, sentimientos o sensaciones, y dejarlos ir. Una sensación de calma surge de no juzgar lo que pasa por la mente o el mundo exterior, y simplemente aceptar la experiencia tal como es (Huppert y Johnson, 2010). Al disminuir la ansiedad se produce a su vez, un aumento en el bienestar psicológico, ya que hay una menor preocupación por eventos futuros (Ricarte et 
al., 2015). Asimismo, la reducción de estrés se puede asociar también con la práctica de prestar atención a la respiración; lo que puede inducir la relajación y disminuir la respuesta fisiológica, un aspecto central en la experiencia emocional (Didonna, 2011); $y$, como consecuencia, aumentar el bienestar (Beauchemin, Hutchins y Patterson, 2008).

En relación con la regulación emocional, ésta aumenta en la mayor parte de los estudios que la evaluaron, con independencia del tipo de diseño. Los resultados apoyan la idea de que las prácticas en atención plena, facilitan la toma de conciencia y la regulación emocional a través de una mayor capacidad de asignar recursos de atención y aprender a situarse en el momento presente (Crescentini et al., 2016). La regulación emocional se relaciona con la aceptación de la respuesta emocional, el control de impulsos, la conciencia corporal, la claridad emocional, y las estrategias de regulación de la emoción (Gratz y Roemer, 2004). A su vez, se asocia con un mayor rendimiento académico y competencia social (Spinrad et al., 2006).

Respecto a la depresión, se observa que los síntomas asociados disminuyen y se mantienen en el tiempo, sobre todo en la etapa de secundaria. Con la práctica de mindfulness los participantes aprenden a distanciarse de los pensamientos y sentimientos; se centran en el momento presente, sin juzgar y con aceptación (Fresco, Segal, Buis y Kennedy, 2007). Este distanciamiento reduce la rumiación y la posibilidad de recaída en la depresión (Didonna, 2011). En el área emocional se percibe, además, una mejoría en los grupos de control activo que realizan alguna actividad basada en emociones, como es el caso del estudio de Crescentini et al. (2016).

Estos resultados en las variables emocionales pueden equipararse a otros estudios realizados con niños y adolescentes que mostraron beneficios en la reducción de estrés, depresión, ansiedad (Beauchemin et al., 2008) y regulación de emociones tras la aplicación de la MBI (Hofmann et al., 2010).

Respecto al bienestar, se encuentran mejoras significativas en todos los estudios que lo evalúan. En un estudio (Terjestam et al., 2016) se observa que en el alumnado de $5^{\circ}$ grado la mejora es más significativa que en $7^{\circ}$ y $8^{\circ}$ curso de secundaria. En estos últimos cursos la $\mathrm{MBI}$ es aplicada por varios profesores, lo que puede dar lugar a diferencias en las prácticas, mientras que en $5^{\circ}$ curso se imparte por un único profesor. En otro estudio (Kuyken et al., 2013) se mantienen los beneficios incluso en momentos de mayor tensión como es la época de exámenes. La MBI puede incrementar, por tanto, la resistencia a estas situaciones estresantes. Un mayor bienestar aumenta las emociones positivas, las relaciones interpersonales satisfactorias y un funcionamiento psicológico positivo.

Al evaluar la atención plena se producen efectos favorables en todos los estudios analizados, con independencia del tipo de grupo de control y programa. Se observan mayores efectos en estudiantes que tienen hogar respecto a estudiantes sin hogar, que viven en un albergue con sus familias; aunque estos informaron de beneficios en su vida cotidiana (Viafora et al., 2015). Una posible explicación es que éstos han sido expuestos más frecuentemente a situaciones traumáticas ambientales estresantes, y por ello experimentan también beneficios (Downer, 2001). La atención plena está relacionada con mayor calidad de vida, competencia académica y habilidades sociales; menor ansiedad, depresión y quejas somáticas (Greco, Baer y Smith, 2011 ). Con las MBI los practicantes alcanzan un estado no crítico de la mente, esto les permite tomar distancia de la experiencia misma, aumentar la capacidad de volver a centrarse en la tarea en cuestión y reducir las reacciones automáticas a estímulos irrelevantes (Hayes y Feldman, 2004). Por tanto, no es de extrañar que la atención cognitiva también mejore.

A su vez, se observa que la competencia social presenta una mayor incidencia en primaria. Los beneficios de la atención plena en las relaciones interpersonales pueden derivarse de aspectos como: responder conscientemente, en lugar de reaccionar automáticamente; mayor conciencia de la conducta y sentimientos de los demás; así 
como, una mayor aceptación de uno mismo da lugar a una mayor empatía y aceptación de los demás (Baer, 2003).

Los resultados en las variables psicosociales están en consonancia con investigaciones anteriores que mostraron mejoras en competencias sociales (Diamond y Lee, 201 1), desempeño académico (Durlak et al., 2011) y bienestar personal (Brown y Ryan, 2003).

En las funciones ejecutivas como la flexibilidad psicológica se observan efectos beneficiosos en algunos de los estudios; sin embargo, existen datos contradictorios que pueden estar motivados por: (a) la aplicación de las $\mathrm{MBI}$ por un instructor externo del centro (Viafora et al., 2015); algunos autores afirman que la práctica de la atención plena a diario, así como la habilidad en la enseñanza son los principales medios para transmitir adecuadamente la práctica (Crane, Kuyken, Hastings, Rothwell, y Williams, 2010; Kabat Zinn, 2003); (b) el origen de la familia; ya que estudiantes de familias de bajos ingresos tienden a exhibir funciones ejecutivas más pobres (Hackman y Farah, 2008); (c) la duración de los programas; los resultados muestran que un mayor número de sesiones se relaciona con mayor flexibilidad cognitiva, así como, mayor memoria de trabajo. Según afirman autores (Burnett, 2011; Ricarte et al., 2015; Terjestam et al., 2016), el éxito de estos programas reside en su práctica a largo plazo.

Estos hallazgos en las variables cognitivas confirman los encontrados en diferentes investigaciones (Napoli, Krech y Holley, 2005; Zylowska et al., 2008). Las prácticas en mindfulness apoyan, por tanto, el desarrollo de las funciones ejecutivas (Zelazo y Lyons, 2012), que se asocian con la activación de diferentes áreas cerebrales (Didonna, 2011 ).

Las $\mathrm{MBI}$ mejoran, por consiguiente, la atención y las funciones ejecutivas, como la flexibilidad cognitiva, al centrar la atención en la respiración y traerla de nuevo cuando esta se aleja, así como, la regulación emocional (Lutz, Slatger, Dunee y Davidson, 2008); e inhiben un procesamiento elaborativo secundario de pensamientos, sentimientos o sensaciones (Bishop et al., 2004). Según los resultados de algunos estudios se confirma que el control inhibitorio es clave en el desarrollo de habilidades sociales y el rendimiento académico (Diamond, 2013; Oberle y Schonert-Reich, 2013).

Esta línea de trabajo ha sido muy fructífera; ahora bien, hay una serie de aspectos que son objeto de crítica. En general, los estudios presentan una muestra amplia; sin embargo, en algunos de ellos se critica el tamaño de muestra relativamente pequeño (Crescentini et al., 2016; Flook et al., 2015); esto cuestiona la medida en que se pueden generalizar los resultados. Se recomendaría un tamaño de muestra amplio y la utilización de diferentes escuelas. Asimismo, en la mayor parte de los estudios existe una ausencia de aleatorización a nivel individual. Esto implica la no independencia de los participantes, lo que podría sesgar las pruebas estadísticas utilizadas para identificar los efectos (SchonertReichl et al., 2015). Se sugiere la utilización de diseños controlados aleatorizados en investigaciones futuras.

En general, los estudios incluyen un gran número y variedad de medidas aplicadas; lo que puede aumentar el riesgo de un error de tipo I (falsos positivos)(Van de Weijer et al., 2014). Se aconseja una mayor homogeneidad y la utilización de un número menor de variables específicamente elegidas (Van de Weijer et al., 2014). A su vez, los maestros y compañeros que evaluaban las diferentes medidas, no eran ciegos a la condición de tratamiento en la mayoría de los estudios (Flook et al., 2015; Schonert-Reichl et al., 2015). Sería conveniente la utilización de informantes $u$ observadores ciegos a las condiciones, así como la diferenciación de la persona que evalúa las pruebas, de la persona que aplica los programas. Además, se observan discrepancias en las medidas entre los informes de los maestros y los autoinformes de los niños (Crescentini et al., 2016). Algunos autores afirman que los individuos suelen ser más exactos auto-informando de la internalización de síntomas que los padres o maestros, y que los profesores tienden a ver la internalización de síntomas como menos problemática (Tandon, Cardeli y Luby, 2009). 
La mitad de los estudios implementaron las $\mathrm{MBI}$ con instructores externos a la escuela. Según un estudio de meta-análisis (Durlak et al., 2011), se muestra que los beneficios de programas de aprendizaje socio-emocional están relacionados con la aplicación de estos por parte de los maestros. En el presente estudio se observa un mayor porcentaje (70\%) de mejoras significativas cuando los profesores aplican los programas de intervención dentro del horario regular de clases. Asimismo, aunque todos los tipos de intervención están basados en programas de mindfulness, algunas actividades son diferentes o se aplican de distinto modo, lo que puede afectar al resultado. A su vez, el número de las sesiones oscila significativamente entre los estudios; y solamente tres de ellos realizaron seguimientos. Se sugieren intervenciones más largas en el tiempo (Ricarte et al., 2015), ya que podrían mejorar la efectividad de los programas; como también realizar seguimientos más prolongados (Kuyken, 2013). Además, solamente tres estudios aluden a la realización de prácticas de ejercicios de meditación formal en casa por parte de los estudiantes. Tal como se ha observado, puede tener una mayor influencia positiva en los resultados (Carmody y Baer, 2008).

Existe una falta de información en la mayoría de los estudios sobre la fidelidad del programa de intervención en mindfulness impartido por los profesores (Kuyken et al., 2013; Raes et al., 2014; Tarrasch et al., 2017; Thierry et al., 2016; Viafora et al., 2015).

Sugerir la incorporación de los padres a la formación del alumnado en la etapa de preescolar, ya que las investigaciones recientes indican un mayor impacto de la formación infantil cuando se combina con formación a padres (Neville et al., 2013). Así como recomendar que estos sean informantes además de los profesores (Van de Weijer et al., 2014).

¿ेHay evidencias suficientes qué incorporar las prácticas de mindfulness en las escuelas tiene efectos beneficiosos? Los datos apoyan que el mindfulness puede tener efectos positivos aplicado en las escuelas. Además, según autores (Metz et al., 2013; Van de Weijer et al., 2014), tanto estudiantes como docentes pueden beneficiarse si éste se incorpora al currículo regular escolar. En algunos colegios en España el mindfulness es parte de una asignatura obligatoria de educación emocional (Sanmartín, 2015). A los maestros les puede ayudar a la reducción de su propio estrés y como resultado, puede influir en la interacción con el alumnado (Gold et al., 2010).

En cuanto a las limitaciones del presente estudio, los artículos revisados no agotan todos los publicados, además existe un posible sesgo de publicación, según el cual los trabajos que no encuentran eficaz el tratamiento tienen más dificultades para que sean publicados.

\section{CONCLUSIONES}

El presente estudio ofrece perspectivas optimistas para futuras investigaciones de las $\mathrm{MBI}$ en el ámbito educativo, así como su posible incorporación al currículo regular.

La atención plena se relaciona con aumento en la calidad de vida, competencia académica y social, así como reducción de problemas de internalización y externalización.

El éxito de estos programas reside en su práctica a largo plazo, tanto en estudiantes como profesorado.

\section{Conflicto de intereses}

Los autores declaran no tener ningún conflicto de intereses.

\section{REFERENCIAS}

* Artículos incluidos en la muestra.

Achenbach, T. M., \& Dumenci, L. (2001). Advances in empirically based assessment: Revised cross-informant syndromes and new DSM-oriented scales for the CBCL, YSR, and TRF: Comment on Lengua, Sadowski, Friedrich, and Fisher (2001). Journal of Consulting and Clinical Psychology, 69(4), 699-702. doi:10.1037/0022006X.69.4.699 
Arguís, R., Bolsas, A. P., Hernández, S., \& Salvador, M. (2010). Programa "AULAS FELICES". Psicología positiva aplicada a la educación. Zaragoza: SATI.

Baer, R. A. (2003). Mindfulness training as a clinical intervention: A conceptual and empirical review. Clinical Psychology: Science and Practice, 10(2), 125-143. doi:10.1093/clipsy.bpg015

Bandura, A. (2006). Guide for constructing self-efficacy scales. Self-efficacy beliefs of adolescents, 5, 307-337.

Beauchemin, J., Hutchins, T. L., \& Patterson, F. (2008). Mindfulness meditation may lessen anxiety, promote social skills, and improve academic performance among adolescents with learning disabilities. Complementary Health Practice Review, 13(1), 34-45. doi:10.1177/1533210107311624

Bishop, S. R., Lau, M., Shapiro, S., Carlson, L., Anderson, N. D., Carmody, J., Segal, Z. V., Abbey, S., Speca, M., Velting, D., \& Devins, G. (2004). Mindfulness: A proposed operational definition. Clinical psychology: Science and practice, $11(3), 230-241$. doi:10.1093/clipsy.bph077

Brown, K. W., \& Ryan, R. M. (2003). The benefits of being present: mindfulness and its role in psychological well-being. Journal of Personality and Social Psychology, 84(4), 822-848. doi:10.1037/00223514.84 .4 .822

Burnett, R. (2011). Mindfulness in schools: Learning lessons from the adults, secular and buddhist. Buddhist Studies Review, 28(1), 79-120. doi:10.1558/bsrv.v28i1.79

Carmody, J., \& Baer, R. A. (2008). Relationships between mindfulness practice and levels of mindfulness, medical and psychological symptoms and well-being in a mindfulnessbased stress reduction program. Journal of behavioral medicine, 31(1), 23-33. doi:10.1007/s10865-007-9130-7

Crane, R. S., Kuyken, W., Hastings, R. P., Rothwell, N., \& Williams, J. M. G. (2010). Training teachers to deliver mindfulnessbased interventions: Learning from the UK experience. Mindfulness, 1(2), 74-86. doi: $10.1007 / s 12671-010-0010-9$

${ }^{*}$ Crescentini, C., Capurso, V., Furlan, S., \& Fabbro, F. (2016). Mindfulness-oriented meditation for primary school children: Effects on attention and psychological wellbeing. Frontiers in Psychology, 7(805), $1-12$. doi:10.3389/fpsyg.2016.00805

Conduct Problems Prevention Research Group (1995). Teacher Social Competence Scale. Recuperado de https://fasttrackproject.org/ techrept/s/sct/

Davidson, M. C., Amso, D., Anderson, L. C., \& Diamond, A. (2006). Development of cognitive control and executive functions from 4 to 13 years: Evidence from manipulations of memory, inhibition, and task switching. Neuropsychologia, 44(11), 2037-2078. doi:10.1016/i. neuropsychologia.2006.02.006

Diamond, A. (2013). Executive functions. Annual review of psychology, 64, 135-168. doi:10.1146/annurevpsych-113011-143750

Diamond, A., Barnett, W. S., Thomas, J. \& Munro, S. (2007). Preschool program improves cognitive control. Science, 318(5855), 1387-1388. doi:10.1126/ science. 1151148

Diamond, A., \& Lee, K. (2011). Interventions shown to aid executive function development in children 4 to 12 years old. Science, 333(6045), 959-964. doi:10.1126/ science. 1204529

Didonna, F. (Ed.). (2011). Manual clínico de mindfulness. Bilbao: Desclée de Brouwer.

Downer, R. T. (2001). Homelessness and its consequences: The impact on children's psychological well-being. New York: Routledge.

Durlak, J. A., Weissberg, R. P., Dymnicki, A. B., Taylor, R. D., \& Schellinger, K. B. (2011). The impact of enhancing students' social and emotional learning: A meta-analysis of school-based universal interventions. Child development, 82(1), 405-432. doi:10.1111/i.1467-8624.2010.01564.x

Felver, J. C., Celis-de Hoyos, C. E., Tezanos, K., \& Singh, N. N. (2016). A systematic review 
of mindfulness-based interventions for youth in school settings. Mindfulness, 7(1), 34-45. doi:10.1007/s12671-015-0389-4

*Flook, L., Goldberg, S. B., Pinger, L., \& Davidson, R. J. (2015). Promoting prosocial behavior and self-regulatory skills in preschool children through a mindfulness-based kindness curriculum. Developmental Psychology, 51 (1), 44-51. doi:10.1037/a0038256

Fresco, D. M., Segal, Z. V., Buis, T., \& Kennedy, S. (2007). Relationship of posttreatment decentering and cognitive reactivity to relapse in major depression. Journal of consulting and clinical psychology, 75(3), 447-455. doi:10.1037/0022-006x.75.3.447

Garon, N., Bryson, S. E., \& Smith, I. M. (2008). Executive function in preschoolers: a review using an integrative framework. Psychological bulletin, 134(1), 31-60. doi:10.1037/00332909.134.1.31

Gioia, G. A., Espy, K. A., \& Isquith, P. K. (2002). Behavior Rating Inventory of Executive Function-Preschool Version (BRIEF-P). Odessa, FL: Psychological Assessment Resources.

Gold, E., Smith, A., Hopper, I., Herne, D., Tansey, G., \& Hulland, C. (2010). Mindfulness-based stress reduction (MBSR) for primary school teachers. Journal of child and family studies, 19(2), 184-189. doi:10.1007/s10826-0099344-0

Goodman, R., Meltzer, H., \& Bailey, V. (1998). The Strengths and Difficulties Questionnaire: A pilot study on the validity of the selfreport version. European child \& adolescent psychiatry, 7(3), 125-130. doi:10.1007/ $\underline{\text { s007870050057 }}$

Gratz, K. L., \& Roemer, L. (2004). Multidimensional assessment of emotion regulation and dysregulation: Development, factor structure, and initial validation of the difficulties in emotion regulation scale. Journal of psychopathology and behavioral assessment, 26(1), 41-54. doi:10.1023/ B: JOBA.0000007455.08539.94

Greco, L. A., Lambert, W., \& Baer, R. A. (2008). Psychological inflexibility in childhood and adolescence: Development and evaluation of the AFQ-Y. Psychological Assessment, 20(2),
93-102. doi:10.1037/1040-3590.20.2.93

Greco, L. A., Baer, R. A., \& Smith, G. T. (2011). Assessing mindfulness in children and adolescents: development and validation of the Child and Adolescent Mindfulness Measure (CAMM). Psychological assessment, 23(3), 606-614. doi:10.1037/a0022819

Hackman, D. A., \& Farah, M. J. (2008). Socioeconomic status and the developing brain. Trends in Cognitive Sciences, 13(2), 65-73. doi:10.1016/i.tics.2008.11.003

Hayes, S. C., Strosahl, K. D., \& Wilson, K. G. (1999). Acceptance and commitment therapy: An experiential approach to behavior change. Nueva York: Guilford Press.

Hayes, A. M., \& Feldman, G. (2004). Clarifying the construct of mindfulness in the context of emotion regulation and the process of change in therapy. Clinical Psychology: science and practice, $17(3), \quad 255-262$. doi:10.1093/ clipsy.bph080

Hick, S. F., \& Bien, T. (2008). Mindfulness y psicoterapia. Barcelona: Kairós.

Hofmann, S. G., Sawyer, A. T., Witt, A. A., \& Oh, D. (2010). The effect of mindfulnessbased therapy on anxiety and depression: $A$ meta-analytic review. Journal of consulting and clinical psychology, 78(2), 169-183. doi:10.1037/a0018555

Huppert, F. A., \& Johnson, D. M. (2010). A controlled trial of mindfulness training in schools: The importance of practice for an impact on well-being. The Journal of Positive Psychology, 5(4), 264-274. doi:10.1080/17439761003794148

Kabat-Zinn, J. (1982). An outpatient program in behavioural medicine for chronic pain patients based on the practice of mindfulness meditation: theoretical considerations and preliminary results. General Hospital Psychiatry, 4(1), 33-47. doi:10.1016/01638343(82)90026-3

Kabat-Zinn, J. (2003) Mindfulness-based interventions in context: past, present, and future. Clinical Psychology: Science and Practice, 10(2), 144-156. doi:10.1093/ clipsy.bpg016

Kristeller J. L., \& Hallett C. B. (1999). An 
exploratory study of a meditation-based intervention for binge eating disorder. Journal of Health Psychology, 4(3), 357-363. doi:10.1177/135910539900400305

*Kuyken, W., Weare, K., Ukoumunne, O. C., Vicary, R., Motton, N., Burnett, R., Cullen, C., Hennelly, S., \&Huppert, F. (2013). Effectiveness of the mindfulness in schools programme: non-randomised controlled feasibility study. The British Journal of Psychiatry, 203(2), 126 131. doi:10.1192/bjp.bp. 113.126649

LaFreniere, P. J., \& Dumas, J. E. (1996). Social competence and behavior evaluation in children ages 3 to 6 years: The short form (SCBE-30). Psychological Assessment, 8(4), 369-377. doi:10.1037//10403590.8.4.369

Lickona, T., \& Davidson, M. (2003). School as a caring community profile-II (SCCP-II). New York: Center for the 4th and 5th Rs., State University of New York at Cortland.

Linehan, M. M. (2003). Manual de tratamiento de los trastornos de personalidad límite. Barcelona: Paidós.

López-González, L., González, M. Á., \& Alzina, R. B. (2016). Mindfulness e investigaciónacción en educación secundaria. Gestación del Programa TREVA. Revista Interuniversitaria de formación del profesorado, 30(3), 75-91.

Lutz, A., Slagter, H. A., Dunne, J. D., \& Davidson, R. J. (2008). Attention regulation and monitoring in meditation. Trends in Cognitive Sciences, 12(4), 163-169. doi:10.1016/i. tics.2008.01.005

Mañas, I., Franco, C., Gil, M., \& Gil, C. (2014). Educación consciente: Mindfulness (Atención Plena) en el ámbito educativo. Educadores conscientes formando a seres humanos conscientes. Alianza de civilizaciones, políticas migratorias y educación, 193-229.

*Metz, S. M., Frank, J. L., Reibel, D., Cantrell, T., Sanders, R., \& Broderick, P. C. (2013). The effectiveness of the learning to BREATHE program on adolescent emotion regulation. Research in Human Development, 10(3), 252-272. doi:10.1080/15427609.2013.8 18488

Miller J. J., Fletcher K., \& Kabat-Zinn J. (1995).
Three-year follow-up and clinical implications of a mindfulness meditation-based stress reduction intervention in the treatment of anxiety disorders. General Hospital Psychiatry, 17(3), 192-200. doi:10.1016/01638343(95)00025-M

Modrego-Alarcón, M., Martínez-Val, L., LópezMontoyo, A., Borao, L., Margolles, R., \& García-Campayo, J. (2016). Mindfulness en contextos educativos: profesores que aprenden y profesores que enseñan mindfulness. Revista Interuniversitaria de Formación del Profesorado, 87(30.3), 31-46. Napoli, M., Krech, P. R., \& Holley, L. C. (2005). Mindfulness training for elementary school students: The attention academy. Journal of Applied School Psychology, $21(1), 99-125$. doi:10.1300/J008v21n01 05

Neville, H. J., Stevens, C., Pakulak, E., Bell, T. A., Fanning, J., Klein, S., \& Isbell, E. (2013). Family-based training program improves brain function, cognition, and behavior in lower socioeconomic status preschoolers. Proceedings of the National Academy of Sciences, 110(29), 12138-12143. doi:10.1073/pnas. 1304437110

Noam, G. G., \& Goldstein, L. S. (1998). The resilience inventory. [Protocolo no publicado]. Oberle, E., \& Schonert-Reichl, K. A. (2013). Relations among peer acceptance, inhibitory control, and math achievement in early adolescence. Journal of Applied Developmental Psychology, 34(1), 45-51. doi:10.1016/i.appdev.2012.09.003

Parkhurst, J. T., \&Asher, S. R. (1992). Peer rejection in middle school: Subgroup differences in behavior, loneliness, and interpersonal concerns. Developmental Psychology, 28(2), 231-241. doi:10.1037/00121649.28 .2 .231

*Raes, F., Griffith, J. W., Van der Gucht, K., \& Williams, J. M. G. (2014). School-based prevention and reduction of depression in adolescents: A cluster-randomized controlled trial of a mindfulness group program. Mindfulness, 5(5), 477-486. doi:10.1007/ s12671-013-0202-1

Ramos, N., Recondo, O., \& Enríquez, H. (2012). 
Practica la inteligencia emocional plena: Mindfulness para regular nuestras emociones. Barcelona: Kairós.

Reitan, R. M. (1992). Trail Making Test: Manual for Administration and Scoring. Tucson, AZ: Reitan Neuropsychology Laboratory.

*Ricarte, J. J., Ros, L., Latorre, J. M., \& Beltrán, M. T. (2015). Mindfulness-based intervention in a rural primary school: Effects on attention, concentration and mood. International Journal of Cognitive Therapy, 8(3), 258-270. doi:10.1521/iict_2015 803

Rieffe, C., Oosterveld, P., Miers, A. C., Terwogt, M. M., \& Ly, V. (2008). Emotion awareness and internalising symptoms in children and adolescents: The Emotion Awareness Questionnaire revised. Personality and Individual Differences, 45(8), 756-761. doi:10.1016/i.paid.2008.08.001

Sanmartín, O. R. (15 de junio de 2015). Meditación en el colegio. El Mundo. Recuperado de http://www.elmundo.es/ espana/2015/06/15/556f439c268e3e9e 438b459a.html

*Schonert-Reichl, K. A., Oberle, E., Lawlor, M. S., Abbott, D., Thomson, K., Oberlander, T. F., \& Diamond, A. (2015). Enhancing cognitive and social-emotional development through a simple-to-administer mindfulnessbased school program for elementary school children: A randomized controlled trial. Developmental Psychology, 51(1), 52-66. doi:10.1037/a0038454

Segal, Z., Williams, J., \& Teasdale, J. (2006). Terapia cognitiva de la depresión basada en la consciencia plena. Un nuevo abordaje para la prevención de las recaídas. Bilbao: Descleé de Brouwer.

Spinrad, T. L., Eisenberg, N., Cumberland, A., Fabes, R. A., Valiente, C., Shepard, S. A., Reiser, M., Losoya, S. H., \& Guthrie, I. K. (2006). Relation of emotion-related regulation to children's social competence: a longitudinal study. Emotion, 6(3), 498-510. doi:10.1037/1528-3542.6.3.498

Tandon, M., Cardeli, E., \& Luby, J. (2009). Internalizing disorders in early childhood: A review of depressive and anxiety disorders.
Child and adolescent psychiatric clinics of North America, 18(3), 593-610. doi:10.1016/i.chc.2009.03.004

*Tarrasch, R., Margalit-Shalom, L., \& Berger, R. (2017). Enhancing visual perception and motor accuracy among school children through a mindfulness and compassion program. Frontiers in Psychology, 8(281). doi:10.3389/fpsyg.2017.00281

Tébar, S., \& Parra, M. (2015). Practicando Mindfulness con el alumnado de tercer curso de Educación Infantil. ENSAYOS, Revista de la Facultad de Educación de Albacete, 30(2), 85-97. doi:10578/8995

*Terjestam, Y., Bengtsson, H., \& Jansson, A. (2016). Cultivating awareness at school. Effects on effortful control, peer relations and well-being at school in grades 5,7 , and 8 . School Psychology International, 37(5), 456469. doi:10.1177/0143034316658321

Terzi, A. M., de Souza, E. L., Machado, M. P. A., Konigsberger, M., Waldemar, J. O. C., de Freitas, B. I., Matarazzo-Neuberger, W. M., Migliori, R. F., Kawamata, R. N., Alvarenga, L. F. R., Ferreira, M. Q. B., \& Demarzo, M. (2016). Mindfulness en la Educación: experiencias y perspectivas desde Brasil. Revista Interuniversitaria de formación del profesorado, 30(3), 107-122.

*Thierry, K. L., Bryant, H. L., Nobles, S. S., \& Norris, K. S. (2016). Two-year impact of a mindfulness-based program on preschoolers' self-regulation and academic performance. Early Education and Development, 27(6), 805-821. doi:10.1080/10409289.2016.1 141616

Urrútia, G., \& Bonfill, X. (2010). Declaración PRISMA: una propuesta para mejorar la publicación de revisiones sistemáticas y metaanálisis. Medicina clínica, 135(11), 507-511.

Vallejo, M. A. (2006). Mindfulness. Papeles del Psicólogo, 27(2), 92-99.

*Van de Weijer-Bergsma, E., Langenberg, G., Brandsma, R., Oort, F. J., \& Bögels, S. M. (2014). The effectiveness of a schoolbased mindfulness training as a program to prevent stress in elementary school children. 
Mindfulness, 5(3), 238-248. doi:10.1007/ s12671-012-0171-9

*Viafora, D. P., Mathiesen, S. G., \& Unsworth, S. J. (2015). Teaching mindfulness to middle school students and homeless youth in school classrooms. Journal of child and family studies, 24(5), 1179-1191. doi:10.1007/ s10826-014-9926-3

Wentzel, K. R. (1993). Motivation and achievement in early adolescence: The role of multiple classroom goals. Journal of Early Adolescence, 13(1), 4-20. doi:10.1177/0272431693013001001

Zelazo, P. D., \& Lyons, K. E. (2012). The potential benefits of mindfulness training in early childhood: A developmental social cognitive neuroscience perspective. Child Development Perspectives, 6(2), 154-160. doi:10.1111/ j.1750-8606.2012.00241.x

Zenner, C., Herrnleben-Kurz, S., \& Walach, H. (2014). Mindfulness-based interventions in schools-a systematic review and metaanalysis. Frontiers in psychology, 5(603), 1-20. doi:10.3389/fpsyg.2014.00603 Zylowska, L., Ackerman, D. L., Yang, M. H., Futrell, J. L., Horton, N. L., Hale, T. S., Pataki, C., Smalley, S. L. (2008). Mindfulness meditation training in adults and adolescents with ADHD: a feasibility study. Journal of Attention Disorders, 11(6), 737-746. doi: $10.1177 / 1087054707308502$

\section{APÉNDICE}

\section{NOTA TABLA 1: ESCALAS DE MEDIDA}

Nota: $\quad$ DASS-21=Depression Anxiety Stress Scales; NPDK=Non-Productive Thoughts Questionnaire for Children; EAQ-30=Emotion Awareness Questionnaire; SOC-K=Sense of Coherence Questionnaire for Children; SHS=Subjective Happiness Scale; SCARED-71=Screen for Child Anxiety Related Emotional Disorders; SCBE$30=$ Social Competence and Behavior Evaluation; SDSC $=$ Sleep Disturbance Scale for Children; SCCP II=School as a Caring Community Profile II; TSC=Teacher Social Competence Scale; TS=Sharing Task; GD=Delay of Gratification; TF=Flanker Task; DCCS=Dimensional Change Card Sort Task; GS=School Grades; STAIC=StateTrait Anxiety Inventory for Children; TMT=Trail Making Test; Faces-R=Test de Percepción de Diferencias-Revisado; WISC-III=Digit Span Test from the Wechsler Intelligence Scale for Children; $A R=$ Ansiedad Rasgo.

\section{NOTA TABLA 2: ESCALAS DE MEDIDA}

Nota: $Q D=$ Demographic Questionnaire; WEMWBS=WarwickEdinburgh Mental Well-Being Scale; PSS=Perceived Stress Scale; QEF=Feedback Evaluation Questionnaire; CES-D $=$ Center for Epidemiologic Studies Depression Scale; DERS=Difficulties in Emotion Regulation Scale; PCS=Psychosomatic Complaints Scale; SS=Stress Scale; ASRES=Affective Self-Regulatory Efficacy Scale;
SAVPS=Survey Acceptability and Perceived Social Validity; $\mathrm{CAMM}=$ The Child Acceptance and Mindfulness Measure; AFQ-Y $=$ The Avoidance and Fusion Questionnaire for Youth; SCS-C=The Self-Compassion Scale for Children; $P E Q=$ Participant Evaluation Questionnaire; TF $=$ Flanker Task; $\mathrm{TFH}=$ Hearts and Flowers Task; CS=Cortisol Salivar; $\mid \mathrm{R} I=$ Interpersonal Reactivity Index modified for children; $\mathrm{RI}=$ Resiliency Inventory; $\mathrm{SDQ}=$ Self-Description Questionnaire; SPQC=Seattle Personality Questionnaire for Children; MAAS-C=Mindful Attention Awareness Scale adapted for children; $S G Q=$ Social Goals Questionnaire; $M P=$ Peer-Report Measures; $M G=$ Math Grades; $C B C L$ TRF $=$ Child Behavior Checklist-Teacher Report Form; CTRS$\mathrm{R}=$ Conners Teachers Rating Scales; $\mathrm{SMFQ}=$ Short Mood and Feelings Questionnaire; EATO-R=Early Adolescence Temperament Questionnaire; EC=Effortful Control Ratings; $W B S=$ Well-Being at School; $P D=$ Psychological Distress; $G S S=$ General Stress Scale; SDQPP $=$ Strengths and Difficulties Questionnaire; BRIEF-P=Inventario de Evaluación Conductual de la Función Ejecutiva-Preescolar; PPVT-4 = Peabody Picture Vocabulary Test-Fourth Edition; $\mid \mathrm{SIP}=$ Early Reading Assessment; $\mathrm{VMI}=$ Beery-Buktenica Developmental Test of Visual-Motor Integration: $\mathrm{VP}=$ Perception Visual, $\mathrm{MC}=$ Motor Coordination; $\mathrm{SCAS}=$ Spence Children's Anxiety Scale; FFMQ =Five Facet Mindfulness Questionnaire. 